\title{
Aarno Perfil das anomalias congênitas em nascidos vivos de Tangará da Serra, Mato Grosso, 2006-2016*
}

doi: 10.5123/S1679-49742018000300017

\author{
Profile of congenital anomalies among live births in the municipality of Tangará da Serra, \\ Mato Grosso, Brazil, 2006-2016
}

\section{Perfil de anomalías congénitas en nascidos vivos en el municipio de Tangará da Serra, Mato Grosso, Brasil, 2006-2016}

\author{
Juliana Herrero da Silva' \\ Ana Cláudia Pereira Terças ${ }^{3}$ \\ Laura Castelo Branco Pinheiro ${ }^{2}$ \\ Giovanny Vinícius Araújo de França ${ }^{4}$ \\ Marina Atanaka ${ }^{5}$ \\ Lavínia Schüler-Faccini² - ๑ orcid.org/0000-0002-2428-0460 \\ 'Prefeitura Municipal de Tangará da Serra, Secretaria Municipal de Saúde, Tangará da Serra, MT, Brasil \\ 2Universidade Federal do Rio Grande do Sul, Departamento de Genética, Porto Alegre, RS, Brasil \\ ${ }^{3}$ Universidade do Estado de Mato Grosso, Faculdade de Ciências Biológicas, Agrárias e da Saúde, Tangará da Serra, MT, Brasil \\ ${ }^{4}$ Ministério da Saúde, Secretaria de Vigilância em Saúde, Brasília, DF, Brasil \\ ${ }^{5}$ Universidade Federal de Mato Grosso, Departamento de Saúde Coletiva, Cuiabá, MT, Brasil
}

\section{Resumo}

Objetivo: descrever as anomalias congênitas (ACs) entre nascidos vivos de mães residentes em Tangará da Serra, MT, Brasil, durante o período 2006-2016. Métodos: estudo descritivo, com dados do Sistema de Informações sobre Nascidos Vivos (Sinasc). Resultados: de 15.689 nascimentos, 77 foram registrados com ACs (4,9/1.000); houve incremento de 80,7\% no registro de AC em 2016, representando 10,3/1.000 nascidos vivos, incluídos cinco casos de microcefalia; a prevalência de AC foi maior entre crianças de mulheres maiores de 35 anos de idade (razão de prevalência [RP] =1,91; intervalo de confiança $\left.\left[\mathrm{IC}_{95 \%}\right] 1,01 ; 3,60\right)$, prematuros $\left(\mathrm{RP}=2,22 ; \mathrm{IC}_{95 \%} 1,26 ; 3,92\right)$ e com baixo peso ao nascer $\left(\mathrm{RP}=3,21 ; \mathrm{IC}_{95 \%}\right.$ 1,86;5,54). Conclusão: observou-se baixa prevalência de AC, possivelmente relacionada ao sub-registro no nascimento; 0 aumento observado em 2016 pode estar relacionado à epidemia de Zika como causa de microcefalia, e à maior atenção dos profissionais de saúde às ACs durante a emergência em Saúde Pública.

Palavras-chave: Anormalidades Congênitas; Nascimento Vivo; Sistemas de Informação; Vigilância em Saúde Pública; Infecção pelo Zika vírus; Microcefalia. 


\section{Introdução}

As anomalias congênitas (ACs) são distúrbios de desenvolvimento de origem pré-natal presentes ao nascimento, e podem ser estruturais (deformidades físicas), funcionais (alterações neuromotoras) ou metabólicas (por exemplo, os erros inatos de metabolismo, fenilcetonúria, entre outros). Suas causas podem ser genéticas, ambientais ou multifatoriais. ${ }^{1,2}$ $\mathrm{Na}$ maioria dos casos, a etiologia permanece desconhecida e as causas ambientais (teratógenos) ainda são pouco estudadas, especialmente em países em desenvolvimento como o Brasil. ${ }^{1,3}$ Estima-se que as anomalias congênitas estão presentes em 2 a 3\% dos nascidos vivos, e que podem chegar a $5 \%$ se as alterações diagnosticadas mais tardiamente, como as anomalias cardíacas, renais e pulmonares, forem contabilizadas. ${ }^{2}$ Entre 1980 e 2007, houve grande redução proporcional dos óbitos infantis secundários devidos a causas infecciosas e respiratórias, enquanto as ACs permaneceram estáveis, sendo estas a segunda causa de mortalidade infantil no Brasil. ${ }^{1,4}$ Semelhante fenômeno foi descrito em outro país latino-americano, o Chile, entre 2001 e 2010.5

\section{A partir de 1999, foi incluído um campo na DNV para registro das $A C s$, o que torna o Sinasc um instrumento importante para monitoramento de ACs no Brasil.}

O Sistema de Informações sobre Nascidos Vivos (Sinasc) foi instituído em 1990 pelo Ministério da Saúde com o objetivo de fornecer dados sobre os nascidos vivos, extraídos da Declaração de Nascido Vivo (DNV) ${ }^{6,7}$ A partir de 1999, foi incluído um campo na DNV para registro das ACs, codificadas conforme 0 Capítulo XVII da $10^{a}$ Revisão da Classificação Estatística Internacional de Doenças e Problemas Relacionados com a Saúde (CID-10), ${ }^{3}$ o que torna o Sinasc um instrumento importante para monitoramento de ACs no Brasil. ${ }^{8}$

Com a emergência nacional causada pelo vírus Zika e sua associação com microcefalia, ${ }^{9}$ o serviço de vigilância epidemiológica do município de Tangará da Serra, Mato Grosso, Brasil, percebeu a importância de ter o registro do perímetro cefálico e do comprimento ao nascer dos nascidos vivos (dados até então ausentes da DNV) e instituiu, em todas as maternidades locais, a obrigatoriedade de seu registro manual a partir de 2016. Em 2017, a iniciativa foi estendida a todo 0 território nacional em atendimento à recomendação do Ministério da Saúde.

$\mathrm{Na}$ busca por compreender o comportamento das anomalias congênitas em Tangará da Serra nos últimos 11 anos (2006-2016), período que antecedeu a emergência da síndrome congênita por Zika (SCZ), bem como durante a ocorrência dessa doença com transmissão continuada do vírus, o presente estudo teve como objetivo descrever as anomalias congênitas em nascidos vivos de mães residentes em Tangará da Serra no período de 2006 a 2016.

\section{Métodos}

Trata-se de um estudo descritivo, com base nos dados da DNV e do Sinasc referentes ao período compreendido entre $1^{\circ}$ de janeiro de 2006 e 31 de dezembro de 2016, consultados no segundo semestre de 2017. Foram incluídos todos os nascidos vivos residentes no município de Tangará da Serra, localizado na região sudoeste mato-grossense, a $240 \mathrm{~km}$ de Cuiabá, capital do estado. A população local, estimada em 2017, era de 98.828 habitantes, com uma média anual de 1.445 nascidos vivos. Tangará da Serra é a principal cidade de sua microrregião e sexta maior de Mato Grosso, com cerca de $3 \%$ da população do estado $;{ }^{10}$ possui 211 estabelecimentos de saúde, públicos ou privados, inscritos no Cadastro Nacional de Estabelecimentos de Saúde (CNES). Entre esses estabelecimentos, encontram-se quatro hospitais, três com maternidade. ${ }^{11}$

Foram incluídos todos os nascidos vivos entre $1^{\circ} \mathrm{de}$ janeiro de 2006 e 31 de dezembro de 2016, com base em dados do Sinasc extraídos em $1^{\circ}$ de setembro de 2017. O Sinasc é organizado a partir dos registros na Declaração de Nascido Vivo, feitos pelo profissional de saúde. Para esta análise, foram selecionadas as seguintes variáveis da DNV:

a) data de nascimento (dia, mês e ano);

b) sexo do nascido vivo (masculino; feminino);

c) peso ao nascer (em gramas: igual ou superior a $2.500 \mathrm{~g}$; inferior a $2.500 \mathrm{~g}$ );

d) idade materna (em anos: menor que 35; 35 ou mais);

e) escolaridade materna (em anos de estudo: até 7; 8 a $11 ; 12$ ou mais); 
f) número de gestações anteriores (nenhuma; uma; duas; três ou mais);

g) número de filhos nascidos vivos (nenhum; um; dois; três ou mais);

h) número de perdas fetais (nenhuma; uma; duas ou mais);

i) duração da gestação (em semanas: menos de 36; 37 a $41 ; 42$ ou mais);

j) tipo de gestação (única; dupla); e

k) presença de anomalias congênitas.

As anomalias congênitas são registradas em um campo especial na DNV. Esse campo permite o registro de uma ou mais anomalias congênitas. Cada diagnóstico deve ser informado tanto no formulário da DNV como no Sinasc. Compete ao médico diagnosticar os defeitos e/ou anomalias, bem como informar a quem preenche e/ou digita a DNV os códigos correspondentes, de acordo com a CID- $10 .^{3}$ 0 registro das informações no hospital pode ser dificultado pelo fluxo inadequado, especialmente quando diferentes pessoas coletam e preenchem os dados da DNV. Por isso, recomenda-se que o preenchimento da DNV seja feito até o momento da alta da mãe e por uma única pessoa. No caso de defeitos congênitos diagnosticados após a emissão da DNV, ou quando a confirmação diagnóstica laboratorial é tardia, é possível registrar o dado como ressalva e acrescentar a informação no banco de dados do Sinasc.

A análise estatística foi realizada utilizando-se 0 software Stata versão 13.0. ${ }^{12}$ Inicialmente, foram verificados os números anuais de nascidos vivos com qualquer anomalia congênita (isolada ou múltiplas) e sua prevalência por mil nascimentos. Logo, foram estimadas as prevalências de ACs (por mil nascimentos) por tipo, de acordo com a CID-10, para todo o período de 2006 a 2016, tendo como numerador o número de nascidos vivos com cada tipo/grupo de $\mathrm{AC}$ e como denominador o total de nascidos vivos no período. Foram calculadas as razões de prevalência (RP) e seus respectivos intervalos de confiança de $95 \%$ $\left(\mathrm{IC}_{95 \%}\right)$, segundo características sociodemográficas, do parto e dos nascidos vivos.

0 estudo foi realizado no âmbito das ações da Vigilância Epidemiológica do município de Tangará da Serra. Foram utilizados apenas dados secundários, sem identificação direta ou indireta das pessoas. Por seguir os padrões éticos definidos nas Resoluções do
Conselho Nacional de Saúde (CNS) n ${ }^{0}$ 466, de 12 de dezembro de 2012, e ${ }^{0} 510$, de 7 de abril de 2016, o projeto do estudo foi dispensado de apreciação por Comitê de Ética em Pesquisa.

\section{Resultados}

Foram analisados 15.689 nascidos vivos de mães residentes no município de Tangará da Serra no período entre 2006 e 2016. Desses, 77 apresentaram alguma $\mathrm{AC}$ identificada até o momento da alta, representando uma prevalência de AC de 4,9/1.000 nascidos vivos.

A Figura 1 apresenta as prevalências do total de ACs entre os anos de 2006 e 2016. Chama a atenção que na década entre 2006 e 2015, os registros se mantiveram estáveis, embora houvesse um aumento de 80,7\% na prevalência de ACs entre 2015 (5,7/1.000) e 2016 (10,3/1.000 nascidos vivos).

O número total de anomalias foi de 90 , uma vez que sete nascidos vivos apresentavam mais de uma anomalia. As ACs do aparelho osteomuscular (27/90) foram as mais frequentes, seguidas pelas do sistema nervoso (23/90), aparelho digestivo (12/90), fenda labial e palatina (6/90) e cromossômicas (4/90). Entre as ACs do sistema osteomuscular, os diagnósticos mais registrados foram 'pé torto' (7/27) e polidactilia (5/27). Dos 23 registros de ACs do sistema nervoso, o diagnóstico mais encontrado foi de microcefalia, com seis registros - cinco deles apenas no ano de 2016 -, seguido de hidrocefalia (5/23), anencefalia (3/23), espinha bífida (2/23) e encefalocele (2/23) (Tabela 1).

A Tabela 2 apresenta a distribuição dos nascidos vivos com ACs por tipo de anomalia e ano de nascimento, incluídas as dez ACs mais frequentes no período. De 2006 a 2015, apenas um caso de microcefalia havia sido registrado no município, enquanto no ano seguinte, 2016, foram notificados nada menos que cinco casos, todos de microcefalia grave.

Quanto às características maternas e dos nascidos vivos, as RP de ACs foram maiores entre os filhos de mulheres com idade superior a 35 anos, em relação àquelas com menos de 35 anos $\left(\mathrm{RP}=1,91 ; \mathrm{IC}_{95 \%}\right.$ $1,01 ; 3,60)$, entre crianças prematuras, na comparação com as nascidas a termo $\left(\mathrm{RP}=2,22 ; \mathrm{IC}_{95 \%} 1,26 ; 3,92\right)$, e naquelas com peso ao nascer inferior a $2.500 \mathrm{~g}$ $\left(\mathrm{RP}=3,21 ; \mathrm{IC}_{95 \%} 1,86 ; 5,54\right)$ (Tabela 3). 


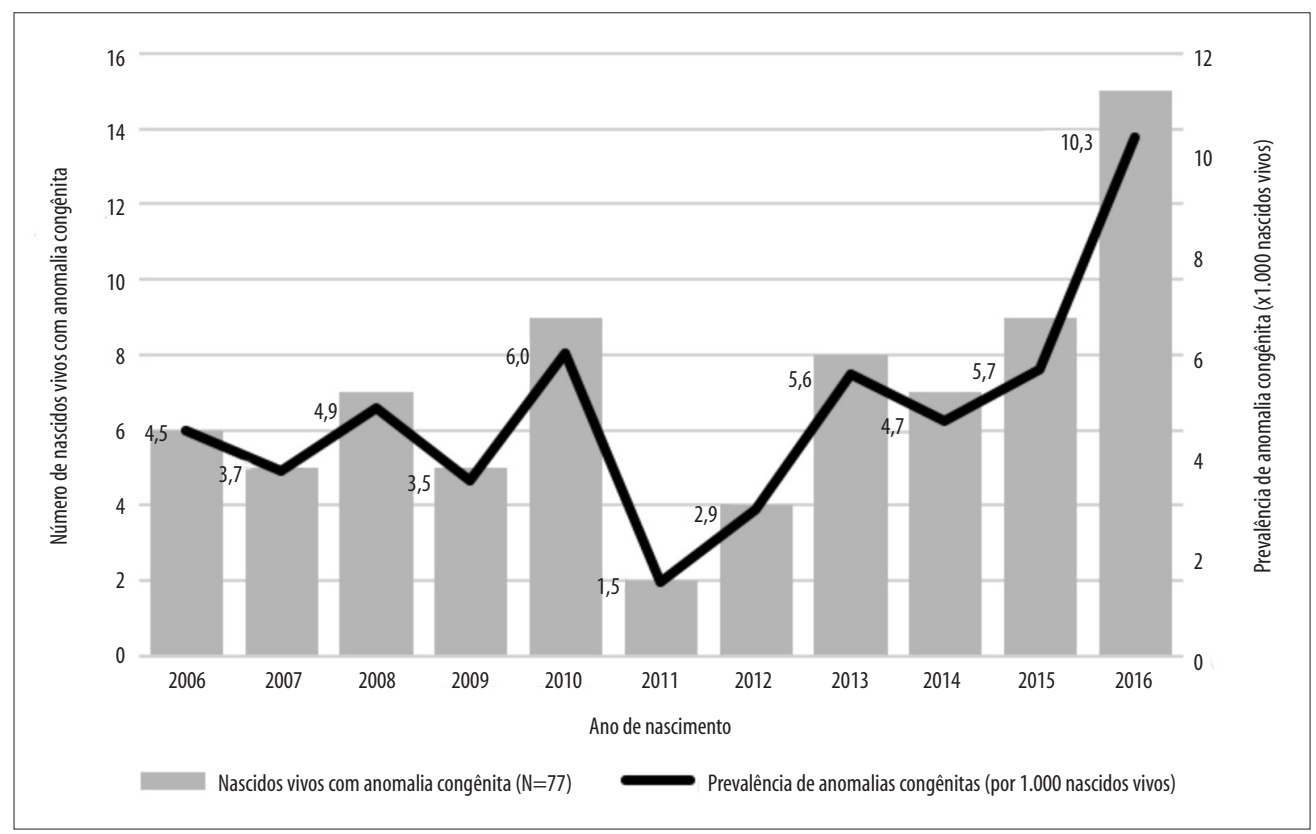

Fonte:Sistema de Informações sobre Nascidos Vivos (Sinasc); dados extraídos em 01/09/2017.

\section{Figura 1 - Número de nascidos vivos com anomalia congênita e prevalência de anomalias congênitas (por 1.000 nascidos vivos), Tangará da Serra, Mato Grosso, 2006-2016}

\section{Discussão}

Foram avaliadas, retrospectivamente, as prevalências anuais de ACs registradas pelo Sinasc, entre 2006 e 2016, em um município no estado do Mato Grosso no Centro-Oeste brasileiro, onde ocorreu um surto de Zika vírus no final do ano de 2015 e início de 2016. A epidemia pelo vírus Zika (ZIKV), reconhecido teratógeno em humanos, enfatizou a importância do estabelecimento de protocolos de vigilância de ACs em países em desenvolvimento. A Organização Mundial da Saúde (OMS) preconiza como responsabilidade de cada país estabelecer protocolos e regulamentos para vigilância de $\mathrm{ACs},{ }^{13}$ responsáveis pelos 276 mil óbitos neonatais precoces por anomalia congênita no mundo, a cada ano. ${ }^{14}$

Na Europa, foi criada em 1979 a European Surveillance of Congenital Anomalies (Eurocat), uma rede de registros populacionais para a vigilância epidemiológica de ACs, com 23 países integrantes. ${ }^{15}$

Nas Américas, existem registros de ACs em diversos países, como Argentina, Brasil, Canadá, Chile, Colômbia, Costa Rica, México e Uruguai, além do Estudo Colaborativo Latino-Americano de Malformações Congênitas (ECLAMC), o qual reúne hospitais de diversos países da América do Sul. Nos Estados Unidos, o apontamento - de base estadual - é obrigatório e o serviço hospitalar repassa os dados de nascimentos com ACs e óbitos fetais por ACs mediante formulário padronizado. A maioria desses dados são reportados a instâncias maiores, como a International Clearinghouse for Birth Defects and Monitoring Systems (ICBDMS), encarregadas de uma vigilância internacional. ${ }^{16}$

No Brasil, a notificação é obrigatória desde 1999 e é feita no preenchimento da DNV; disponibilizados no Sinasc, seus dados permitem a monitoração das ACs. ${ }^{6,7}$

Em Mato Grosso, a cobertura do Sinasc entre os anos 2000 e 2012 foi de $94,9 \%$, considerada satisfatória e com tendência de aumento ${ }^{17}$ acima da média do Brasil, de $92 \%{ }^{4}$

A frequência estimada de ACs é de 2 a 3\% em nascidos vivos. 0 ECLAMC registra 2,73\% dos nascidos vivos na América Latina com ACs, enquanto nos países europeus cobertos pela rede EUROCAT, essa média é de 9,5/1.000 nascimentos vivos e de 0,9/1.000 óbitos perinatais. Essas diferenças entre as frequências na América Latina e na Europa devem-se, em grande parte, à realização eletiva de interrupções da gestação (abortos eletivos) por anomalias fetais. Quando os registros incluem essas anomalias, a frequência de ACs chega a $2 \%$ nos países europeus. ${ }^{2,14}$ 
Tabela 1 - Distribuição e prevalência dos tipos de malformações congênitas ao nascer segundo a 10ª Revisão da Classificação Estatística Internacional de Doenças e Problemas Relacionados à Saúde, Tangará da Serra, Mato Grosso, 2006-2016

\begin{tabular}{|c|c|c|}
\hline \multirow[t]{2}{*}{ Grupo de doença } & $\begin{array}{l}\text { Nascidos vivos } \\
\text { com anomalias } \\
\text { congênitas } \\
\end{array}$ & $\begin{array}{l}\text { Prevalência de anomalias } \\
\text { congênitas ao nascer } \\
\text { (por } 1.000 \text { nascidos vivos) }\end{array}$ \\
\hline & \multicolumn{2}{|c|}{$n$} \\
\hline Malformações congênitas e deformidades do aparelho osteomuscular & 27 & 1,72 \\
\hline 'Pé torto' & 7 & 0,45 \\
\hline Polidactilia não especificada & 5 & 0,32 \\
\hline Malformações congênitas não especificadas de membro & 3 & 0,19 \\
\hline Gastrosquise & 3 & 0,19 \\
\hline Deformidade congênita não especificada & 3 & 0,19 \\
\hline Hérnia diafragmática congênita & 1 & 0,06 \\
\hline Outras deformidades congênitas do pé & 1 & 0,06 \\
\hline Ausência congênita da mão e de dedo(s) & 1 & 0,06 \\
\hline Nanismo tanatofórico & 1 & 0,06 \\
\hline Outras deformidades congênitas do crânio, da face e da mandíbula & 1 & 0,06 \\
\hline Sindactilia não especificada & 1 & 0,06 \\
\hline Malformações congênitas do sistema nervoso & 23 & 1,47 \\
\hline Microcefalia & 6 & 0,38 \\
\hline Hidrocefalia congênita não especificada & 5 & 0,32 \\
\hline Anencefalia & 3 & 0,19 \\
\hline Espinha bífida não especificada & 2 & 0,13 \\
\hline Outras síndromes com malformações congênitas com outras alterações do esqueleto & 2 & 0,13 \\
\hline Encefalocele não especificada & 2 & 0,13 \\
\hline Holoprosencefalia & 1 & 0,06 \\
\hline Síndrome de Arnold-Chiari & 1 & 0,06 \\
\hline Hipoplasia e displasia da medula espinal & 1 & 0,06 \\
\hline Malformações congênitas do aparelho digestivo & 12 & 0,76 \\
\hline Malformações congênitas dos lábios & 5 & 0,32 \\
\hline Ausência, atresia e estenose congênita & 2 & 0,13 \\
\hline Malformação congênita não especificada & 1 & 0,06 \\
\hline Outras malformações congênitas da boca & 1 & 0,06 \\
\hline Ausência, atresia e estenose congênita & 1 & 0,06 \\
\hline Malformação congênita não especificada & 1 & 0,06 \\
\hline Atresia de esôfago com fístula traqueoesofágica & 1 & 0,06 \\
\hline Fenda labial e fenda palatina & 6 & 0,38 \\
\hline Fenda palatina não especificada & 3 & 0,19 \\
\hline Fenda do palato com fenda labial & 3 & 0,19 \\
\hline Malformações congênitas do aparelho circulatório & 4 & 0,25 \\
\hline Malformação não especificada no coração & 2 & 0,13 \\
\hline Comunicação interventricular & 1 & 0,06 \\
\hline Síndrome do coração esquerdo hipoplásico & 1 & 0,06 \\
\hline Outras malformações congênitas & 4 & 0,25 \\
\hline Malformações congênitas múltiplas, não classificadas em outra parte & 2 & 0,13 \\
\hline Síndromes com malformações congênitas afetando predominantemente 0 aspecto da face & 1 & 0,06 \\
\hline Outras malformações congênitas especificadas do intestino & 1 & 0,06 \\
\hline
\end{tabular}




\section{Tabela 1 - Distribuição e prevalência dos tipos de malformações congênitas ao nascer segundo a 10ª Revisão da Classificação Estatística Internacional de Doenças e Problemas Relacionados à Saúde, Tangará da Serra, Mato Grosso, 2006-2016}

\begin{tabular}{lcc}
\hline Grupo de doença & $\begin{array}{c}\text { Nascidos vivos } \\
\text { com anomalias } \\
\text { congênitas }\end{array}$ & $\begin{array}{c}\text { Prevalência de anomalias } \\
\text { congênitas ao nascer } \\
\text { (por 1.000 nascidos vivos) }\end{array}$ \\
\cline { 2 - 3 } & $\mathbf{n}$ & \\
\hline Anomalias cromossômicas, não classificadas em outra parte & 4 & 0,25 \\
\hline Síndrome de Down não especificada & 4 & 0,25 \\
\hline Malformações congênitas do aparelho osteomuscular & 4 & 0,25 \\
\hline Outras malformações congênitas do aparelho osteomuscular & 2 & 0,13 \\
Ausência congênita e hipoplasia da artéria umbilical & 1 & 0,06 \\
Malformação congênita não especificada & 1 & 0,06 \\
\hline Malformações congênitas do aparelho respiratório & 3 & 0,19 \\
\hline Hipoplasia e displasia do pulmão & 2 & 0,13 \\
Fissura, entalhe ou fenda nasal & 1 & 0,06 \\
\hline Malformações congênitas do aparelho urinário & 1 & 0,06 \\
\hline Agenesia renal não especificada & 1 & 0,06 \\
\hline Malformações congênitas do olho, do ouvido, da face e do pescoço & 0,06 \\
\hline Malformação congênita não especificada & 1 & 0,06 \\
\hline Malformações congênitas do órgão genital & 1 & 0,06 \\
\hline Hipospádia peniana & 1 & 0,06 \\
\hline
\end{tabular}

Fonte: Sistema de Informações sobre Nascidos Vivos (Sinasc); dados extraídos em 01/09/2017.

No Brasil, anualmente, são registrados em torno de 2,9 milhões de nascidos vivos e espera-se que aproximados 90 mil apresentem algum tipo de $\mathrm{AC}$, se consideramos a prevalência estimada de $3 \%$ para a espécie humana. ${ }^{6}$ Entretanto, estudos baseados nos dados do Sinasc nos municípios do Rio de Janeiro, RJ, e de São Paulo, SP, apresentaram, respectivamente, frequência de 1,7\% e 1,6\% de AC nos nascidos vivos, ${ }^{6,18}$ valores superiores ao encontrado no presente estudo. Em Maringá, PR, e no Vale do Paraíba paulista, os dados se assemelham: respectivamente $0,7 \%$ e $0,76 \%$ de AC em nascidos vivos, próximos do observado neste trabalho, de $0,7 \cdot{ }^{19,20}$ A questão do sub-registro de AC na DNV já foi investigada por Luquetti e Koifmann ${ }^{8}$ em oito hospitais de João Pessoa, PB, Salvador, BA, São Paulo e Campinas, SP, Belo Horizonte, MG, e Florianópolis e Joinville, SC. ${ }^{8}$ Em seu trabalho, no qual foi adotado como padrão ouro o ECLAMC, as autoras observaram, nesses hospitais, sub-registro de pelo menos $40 \%$ das ACs no Sinasc, nos anos de 2004 e 2007. Costa et al. $(2008)^{18}$ também realizaram estudo de comparação do Sinasc com registros hospitalares dos nascidos vivos no estado do Rio de Janeiro no ano de 2004 e encontraram variabilidade de concordância entre 0
Sinasc e os registros hospitalares, dependendo do tipo de AC, sendo as taxas de concordância maiores em ACs do sistema osteomuscular.

Em Tangará da Serra, pode-se atribuir o sub-registro, principalmente, à falta de detecção de ACs de órgãos internos, como cardiopatias congênitas ${ }^{21}$ ou outras cujas características clínicas sejam menos visíveis ao nascimento.$^{22} 0$ predomínio de anomalias congênitas do sistema osteomuscular pode estar relacionado ao fato de incluir anomalias frequentes, como 'pé torto' e polidactilia, ademais de a maioria das anomalias desse sistema ser facilmente visível ao nascimento. ${ }^{2,6}$

Chama a atenção o aumento de ACs no ano de 2016, com a ocorrência de cinco nascimentos de crianças com microcefalia, comparativamente a apenas um caso registrado em todos os anos anteriores. Este resultado poderia decorrer de um maior registro após a incorporação da medida do perímetro cefálico na DNV. Entretanto, os cinco casos notificados eram de microcefalias graves, observáveis clinicamente, mesmo sem a ajuda de fita métrica. Posteriormente, esses cinco casos foram todos avaliados e registrados como compatíveis com a síndrome congênita de Zika. Esta observação sincroniza com a 
Tabela 2 - Número de nascidos vivos com anomalia congênita, por tipo de anomalia e ano de nascimento, Tangará da Serra, Mato Grosso, 2006-2016

\begin{tabular}{lcccccccccccc}
\hline Tipo de anomalia congênita & 2006 & 2007 & 2008 & 2009 & 2010 & 2011 & 2012 & 2013 & 2014 & 2015 & 2016 & Total \\
\hline Pé torto & - & - & 1 & - & 1 & 1 & - & 2 & 1 & - & 1 & 7 \\
Microcefalia & - & - & - & - & - & - & - & - & 1 & - & 5 & 6 \\
Malformaçóes dos lábios & - & - & 1 & 1 & 1 & - & - & - & - & 2 & - & 5 \\
Polidactilia & 2 & - & - & - & - & - & - & - & 1 & - & 2 & 5 \\
Hidrocefalia congênita & - & 1 & - & - & 1 & - & - & - & 1 & - & 1 & 4 \\
Malformaçóes de membro & - & - & 1 & - & - & - & 1 & - & - & 1 & 1 & 4 \\
Síndrome de Down & - & 1 & - & 2 & - & - & - & 1 & - & - & - & 4 \\
Fenda palatina & 1 & - & - & - & 1 & - & - & - & 1 & - & - & 3 \\
Anencefalia & - & - & - & - & - & - & - & 1 & 1 & 1 & - & 3 \\
Gastrosquise & 1 & 1 & 1 & - & - & - & - & - & - & - & - & 3 \\
\hline
\end{tabular}

Fonte:Sistema de Informaçōes sobre Nascidos Vivos (Sinasc); dados extraídos em 01/09/2017. Nota: A tabela inclui as dez anomalias congênitas mais frequentes no período de 2006 a 2016.

ocorrência do surto infeccioso por ZIKV no município, entre o final de 2015 e o início de 2016. No Brasil, os casos de microcefalia mantiveram-se estáveis de 2000 a 2014, com prevalência de 2/10 mil nascidos vivos, alterando-se significativamente a partir de 2015, com 54,6/10 mil nascidos vivos. ${ }^{7}$ Parte desse aumento é atribuída à adoção de critérios de inclusão de microcefalia mais amplos, como o perímetro cefálico entre -2 e -3 escores Z. Outrossim, a medida de perímetro cefálico é muitas vezes sujeita a viés, seja por erros na aferição, seja porque é realizada antes de a criança completar 24 horas de vida.$^{23}$ Não obstante tais obliquidades, a utilização de dados do Sinasc foi fundamental para a confirmação de um aumento anormal e súbito de casos de microcefalia no Nordeste, no final de 2015. ${ }^{7}$ Em Mato Grosso como um todo, entre as semanas epidemiológicas 45/2015 e 35/2017, o estado registrou 386 notificações de casos de microcefalia, dos quais $19 \%$ confirmados como casos de síndrome de Zika congênita. ${ }^{24}$

No presente estudo, a razão de prevalência de ACs foi maior em nascidos vivos de mães com mais de 35 anos de idade. Trata-se de um efeito esperado, pois a idade materna é o principal fator de risco para anomalias cromossômicas. ${ }^{2,6,17}$ É também importante observar que foram registrados três casos de gastrosquise, anomalia mais frequente nos filhos de mães mais jovens. Mais ainda: aparentemente, existe uma tendência temporal mundial de aumento dessa $\mathrm{AC}$ a partir dos anos 1980, confirmada em registros norte-americanos. ${ }^{25}$ Peso inferior a $2.500 \mathrm{~g}$ e prematuridade também foram fatores associados às anomalias congênitas, o que era de se esperar: defeitos congênitos, com frequência, são associados a esses desfechos. ${ }^{3,6,8}$

Como limitações adicionais do presente estudo, destaca-se a incompletude no preenchimento da DNV e a indisponibilidade de exames complementares na assistência pré-natal, para diagnóstico precoce das ACs.

Considerando-se a carência de estudos sobre ACs no Brasil, principalmente na região Centro-Oeste, os resultados apresentados por esta pesquisa poderão contribuir no planejamento das ações em Saúde Pública, direcionar e aprofundar discussões no que concerne à ampliação de ações nas políticas públicas para as ACs e todos os atores envolvidos nesse processo: familiares, amigos, profissionais e sociedade geral. Entre essas ações, destaca-se a importância do treinamento e conscientização dos profissionais quanto ao preenchimento da DNV, cujos dados sobre ACs, uma vez incluídos no Sinasc, constituem importante ferramenta para detecção de alterações nas frequências temporais ou espaciais das anomalias congênitas, a exemplo da ocorrência de microcefalia por infecção materna pelo vírus Zika.

\section{Agradecimentos}

À equipe da Vigilância Epidemiológica da Secretaria Municipal de Saúde de Tangará da Serra: Zulema Salete Dengo Nuernberg, Maria Ozana Ferreira da Silva e Gisele Barbosa da Silva. Os autores agradecem aos orgãos financiadores: Ministério da Saúde e CNPq. 
Tabela 3 - Frequência de nascidos vivos e prevalência de anomalia congênita ao nascer, segundo características sociodemográficas, do parto e dos nascidos vivos, Tangará da Serra, Mato Grosso, 2006-2016

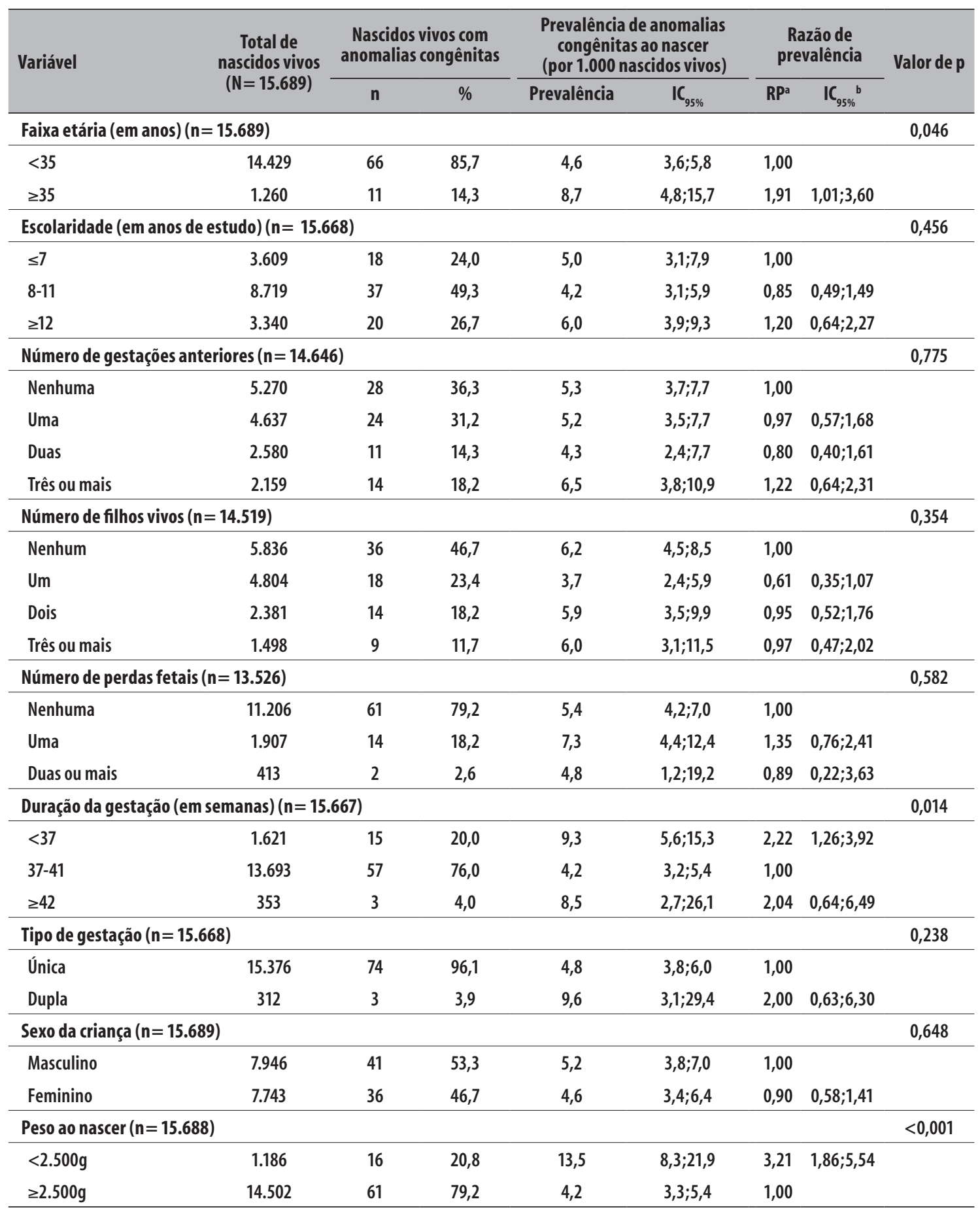

a) RP: razão de prevalência.

b) $\mathrm{IC}_{95 \%}$ : intervalo de confiança de $95 \%$.

Fonte:Sistema de Informações sobre Nascidos Vivos (Sinasc); dados extraídos em 01/09/2017. 


\section{Contribuição dos autores}

Silva JH e Terças ACP participaram da concepção do estudo, coleta, análise e interpretação dos dados, e da redação do manuscrito. Pinheiro LCB, França GVA, Atanaka M e Schüler-Faccini L participaram

\section{Referências}

1. Mazzu-Nascimento T, Melo DG, Morbioli GG, Carrilho E, Vianna FSL, Silva AA, et al. Teratogens: a public health issue - a Brazilian overview. Genet Mol Biol. 2017 Apr-Jun;40(2):387-97.

2. Ramos AP, Oliveira MND, Cardoso JP. Prevalência de malformações congênitas em recém-nascidos em hospital da rede pública. Rev Saúde Com. 2008 janjun;4(1):27-42.

3. Organização Mundial da Saúde. CID-10: classificações internacionais em português. São Paulo: Editora da Universidade de São Paulo; 2014.

4. Victora CG, Aquino EM, Carmo Leal M, Monteiro CA, Barros FC, Szwarcwald CL. Maternal and child health in Brazil: progress and challenges. Lancet. 2011 May;377(9780):1863-76.

5. Canals A, Cavada G, Nazer J. Factores de riesgo de ocurrencia y gravedad de malformaciones congénitas. Rev Méd Chile. 2017 nov;142(11):1431-9.

6. Cosme HW, Lima LS, Barbosa LG. Prevalence of congenital anomalies and their associated factors in newborns in the city of São Paulo from 2010 to 2014. Rev Paul Pediatr. 2017 jan-mar;35(1):33-8.

7. Marinho F, Araujo VEM, Porto DL, Ferreira HL, Coelho MRS, Lecca RCR, et al. Microcephaly in Brazil: prevalence and characterization of cases from the Information System on Live Births (Sinasc), 2000-2015. Epidemiol Serv Saúde. 2016 Oct-Dec;25(4):701-12.

8. Luquetti DV, Koifman RJ. Quality of birth defect reporting in the Brazilian Information System on Live Births (SINASC): a comparative study of 2004 and 2007. Cad Saúde Pública. 2010 Sep;26(9):1756-65.

9. Schuler-Faccini L, Ribeiro EM, Feitosa IML, Horovitz DG, Cavalcanti DP, Pessoa A, et al. Possible association between zika virus infection and microcephaly Brazil, 2015. MMWR Morb Mortal Wkly Rep. 2016 Jan;65(3):59-62.

10. Instituto Brasileiro de Geografia e Estatística. IBGE cidades: Tangará da Serra - MT [Internet]. 2017 [citado 2018 jun 5]. Disponível em: https://cidades. ibge.gov.br/brasil/mt/tangara-da-serra/panorama da análise, interpretação dos dados e redação do manuscrito. Todos os autores participaram de revisão crítica relevante do conteúdo intelectual do manuscrito, aprovaram sua versão final e declaram ser responsáveis por todos os aspectos do trabalho, garantindo sua precisão e integridade.

11. Ministério da Saúde (BR). Cadastro Nacional de Estabelecimento de Saúde (CNES). Tabnet: Brasil [Internet]. 2017 [citado 2018 jun 5]. Disponível em: http://cnes.datasus.gov.br/

12. StataCorp. Stata statistical software: release 7.0. College Station (TX): Stata Corporation; 2013.

13. World Health Organization. Centers for Disease Control and Prevention. Birth defects surveillance: a manual for programme managers [Internet]. Geneva: World Health Organization; 2014 [citado 2018 jun 5]. 126 p. Available in: http://apps.who.int/iris/bitstream/ handle/10665/110223/9789241548724_eng. pdf?sequence $=1$

14. Organización Mundial de la Salud. Anomalías congénitas. Nota descriptiva ${ }^{\circ} 370$ [Internet]. 2015 [cited 2016 Oct 21]. Disponible: http://www.who.int/ es/news-room/fact-sheets/detail/congenital-anomalies

15. European surveillance of congenital anomalies. EUROCAT special report: geographic inequalities in public health indicators related to congenital anomalies [Internet]. Northern Ireland: European surveillance of congenital anomalies; 2014 [cited 2018 jun 5]. 31 p. Available in: http://www.eurocat-network.eu/content/ Special-Report-Geographical-Inequalities.pdf

16. International Clearinghouse for Birth Defects and Monitoring Systems. Addressess and staff [Internet]. 2017 [cited 2018 Jun 5]. Available in: http://www. icbdsr.org/members-address-and-staff/

17. Stevanato JM, Gaíva MAM, Silva AMC. Coverage trends of the Information System on Live Births in Mato Grosso, Brazil, 2000 to 2012. Epidemiol Serv Saude. 2017 Apr-Jun;26(2):265-74.

18. Costa CM, Gama SG, Leal MC. Congenital malformations in Rio de Janeiro, Brazil: prevalence and associated factors. Cad Saúde Pública. 2006 Nov;22(11):2423-31.

19. Melo WA, Zurita RCM, Uchimura TT, Marcon SS. Anomalias congênitas: fatores associados à idade materna em município sul brasileiro, 2000 a 2007. Rev Eletr Enf. 2010 jan-mar;12(1):73-82. 
20. Pinto CO, Nascimento LFC. Estudo de prevalência de defeitos congênitos no Vale do Paraíba Paulista. Rev Paul Pediatr. 2007 set;25(3):233-9.

21. Pinto-Júnior VC, Branco KMPC, Cavalcante RC, Carvalho-Junior W, Lima JRC, et al. Epidemiology of congenital heart disease in Brazil. Rev Bras Cir Cardiovasc. 2015 Mar-Apr;30( 2 ):219-24.

22. Nhoncanse GC, Germano CMR, Avó LRS, Melo DG. Maternal and perinatal aspects of birth defects: a case-control study. Rev Paul Pediatr. 2014 Mar;32(1):24-31.

23. Ministério da Saúde (BR). Secretaria de Vigilância em Saúde. Secretaria de Atenção à Saúde. Orientações integradas de vigilância e atenção à saúde no âmbito da Emergência de Saúde Pública de Importância Nacional: procedimentos para o monitoramento das alterações no crescimento e desenvolvimento a partir

\begin{abstract}
Objective: to describe congenital anomalies (CA) among live births of mothers resident in Tangará da Serra, MT, Brazil, during the period 2006-2016. Methods: this was a descriptive study, using Brazilian Live Birth Information System (SINASC) data. Results: out of 15,689 births, 77 were registered with CA (prevalence of $4.9 / 1,000$ ); there was an $80.7 \%$ increase of recorded $C A$ in 2016, accounting for 10.3/1,000 live births, including five cases of microcephaly; CA prevalence was higher among children born to women aged over 35 years (prevalence ratio $[P R]=1.91$; confidence interval [95\%CI] 1.01;3.60), preterm $(P R=2.22 ; 95 \%$ CI 1.26;3.92) and low birth weight infants (PR=3.21;95\%CI 1.86;5.54). Conclusion: low CA prevalence was found, possibly related to under-recording at birth; the increase observed in 2016 may be related to the Zika epidemic causing microcephaly, as well as greater attention by bealth professionals in relation to $C A$ during this public bealth emergency.

Keywords: Congenital Anomalies; Live Birth; Information Systems; Public Health Surveillance; Zika Virus Infection; Microcephaly.
\end{abstract}

da gestação até a primeira infância, relacionadas à infecção pelo vírus Zika e outras etiologias infeciosas dentro da capacidade operacional do SUS [Internet]. Brasília: Ministério da Saúde; 2017 [citado 2018 jun 5]. 158 p. Disponível em: http://portalarquivos.saude. gov.br/images/pdf/2016/dezembro/12/orientacoesintegradas-vigilancia-atencao.pdf

24. Secretaria Estadual de Saúde (MT). Casos acumulativos até a Semana Epidemiológica 35/2017 - Monitoramento dos casos com alterações relacionadas ao Zika vírus e outras etiologias infecciosas em Mato Grosso - Semana Epidemiológica (SE) 35. Bol Epidemiol. 2017;(31):1-7.

25. St Louis AM, Kim K, Browne ML, Liu G, Liberman RF, Nembhard WN, et al. Prevalence trends of selected major birth defects: a multi-state population-based retrospective study, United States, 1999 to 2007. Birth Defects Res. 2017 Nov;109(18):1442-50.

\section{Resumen}

Objetivo: describir las anomalias congénitas (AC) entre nacidos vivo de madres residentes en Tangara da Serra, MT, Brasil, en el periodo 2006-2016. Métodos: estudio descriptivo, con datos registrados en el Sistema de Informaciones sobre Nacidos Vivos (Sinasc). Resultados: de 15.689 nacimientos del período, 77 se registraron con AC (prevalencia de 4,9/1.000); bubo incremento del 80,7\% de AC en 2016 con 10,3/1.000, inclusos cinco casos de micocefalia; las prevalencias fueron mayores entre hijos de mujeres mayores de 35 años de edad (razón de prevalencia $[R P]=1,91$; intervalo de confianza $\left.\left[I C_{95 \%}\right] 1,01 ; 3,60\right)$, prematuros $(R P=2,22$; $\left.I C_{95 \%} 1,26 ; 3,92\right)$ y con bajo peso al nacer $\left(R P=3,21 ; I C_{95 \%}\right.$ 1,86;5,54). Conclusión: se observó baja prevalencia de $A C$, posiblemente relacionada a los sub-registros de nacimiento; el aumento observado en 2016 puede estar relacionado con la epidemia de Zika como causa de microcefalia, y a la mayor atención de los profesionales de salud en relación a las $A C$ durante la emergencia en salud pública.

Palabras-clave: Anomalías Congénitas; Nascimiento Vivo; Sistemas de Información; Vigilancia en Salud Pública; Infección por el Virus Zika; Microcefalia.

Recebido em 31/01/2018

Aprovado em 04/05/2018 